STRUCTURAL BIOLOGY COMMUNICATIONS

ISSN 2053-230X

\section{A thermostable and alkaline GDSL-motif esterase from Bacillus sp. K91: crystallization and X-ray crystallographic analysis}

\author{
Junmei Ding, ${ }^{a} \ddagger$ Hujie Zhu, ${ }^{a} \ddagger$ Yujia Ye, ${ }^{b}$ Jie Li, ${ }^{c}$ Nanyu Han, ${ }^{a}$ Qian Wu, \\ Zunxi Huang $^{\mathrm{a} *}$ and Zhaohui Meng ${ }^{\mathrm{b} *}$
}

Received 1 October 2017

Accepted 6 January 2018

Edited by A. Nakagawa, Osaka University, Japan

₹ JD and $\mathrm{HZ}$ contributed equally to this work.

Keywords: Est8; crystallization; X-ray crystallographic analysis; GDSL-motif esterase.

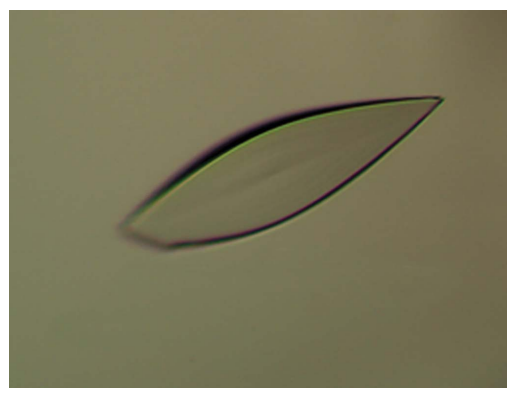

OPEN $\odot$ ACCESS

\begin{abstract}
${ }^{a}$ Engineering Research Centre of Sustainable Development and Utilization of Biomass Energy, Ministry of Education, Yunnan Normal University, 768 Juxian Road, Kunming, Yunnan 650500, People's Republic of China, 'baboratory of Molecular Cardiology, Department of Cardiology, The First Affiliated Hospital of Kunming Medical University, 1168 Chunrong West Road, Kunming, Yunnan 650500, People's Republic of China, and 'Fudan University Shanghai Centre, Institute of Biomedical Science, Fudan University, 131 Dong'an Road, Shanghai 200032, People's Republic of China.*Correspondence e-mail: huangzunxi@163.com, zhhmeng@aliyun.com
\end{abstract}

The esterase Est 8 from the thermophilic bacterium Bacillus sp. K91 belongs to the GDSL family and is active on a variety of acetylated compounds, including 7-aminocephalosporanic acid. In contrast to other esterases of the GDSL family, the catalytic residues Asp182 and His185 were more pivotal for the catalytic activity of Est 8 than the Ser11 residue. To better understand the biochemical and enzymatic properties of Est8, recombinant Est8 protein was purified and crystallized. Crystals of Est8 were obtained by the hanging-drop vapourdiffusion method using $2.0 \mathrm{M}$ ammonium sulfate, 5\%(v/v) 2-propanol as the crystallization solution. X-ray diffraction data were collected to a resolution of $2.30 \AA$ with an $R_{\text {merge }}$ of $16.4 \%$ from a crystal belonging to space group $P 4{ }_{1} 2{ }_{1} 2$ or $P 4_{3} 2{ }_{1} 2$, with unit-cell parameters $a=b=68.50, c=79.57 \AA$.

\section{Introduction}

The esterases (EC 3.1.1.1) and lipases (EC 3.1.1.3) are collectively known as lipolytic enzymes, and have important physiological and biotechnological roles in the synthesis or hydrolysis of ester-containing compounds (Rubin, 1994). Although there are similarities in their molecular structures and catalytic mechanisms, esterases can be distinguished from true lipases by their preference for fatty-acid esters with acylchain lengths of less than ten $\mathrm{C}$ atoms, whereas lipases display maximal activity towards water-insoluble long-chain triglycerides (Jaeger \& Eggert, 2002). Common esterases possess the highly conserved motif Gly- $X$-Ser- $X$-Gly, which contains the catalytic Ser residue (López-López et al., 2014; Stergiou et al., 2013).

As important biocatalysts, lipolytic enzymes are currently attracting enormous attention owing to their numerous applications in industry, such as in the food, leather, fine chemical, detergent, cosmetics, pharmaceutical and paper industries. Although lipolytic enzymes exist in various species, the most widely exploited enzymes are produced by microorganisms (Bornscheuer, 2002). Among them, thermostable lipolytic enzymes are always in great demand because most industrial lipolytic hydrolase and esterification reactions take place under harsh conditions such as at high temperature or under organic solvent conditions. They are better suited to the harsh processes involved in industrial and biotechnological applications (Hess et al., 2008; Hotta et al., 2002).

Recently, we have cloned and characterized a thermostable esterase gene est8 from Bacillus sp. K91 (accession 
No. KJ131182; Ding et al., 2014). A comparison of its aminoacid sequence showed that Est 8 could be grouped into the GDSL family. The GDSL-like (Pfam PF00657) subfamily is characterized by the presence of a distinct GDSL motif that differs from the classical GXSXG motif found in many lipolytic enzymes (Akoh et al., 2004). Est8 can be further classified as a member of the SGNH hydrolase superfamily because of the presence of four strictly conserved and functionally important residues, Ser, Gly, Asn and His, in conserved blocks I, II, III and V, respectively (Mølgaard et al., 2000). Recombinant Est8 was identified as an esterase which prefers to hydrolyze short $p$-nitrophenyl esters of acetate $\left(\mathrm{C}_{2}\right)$. Est8 displayed maximum activity around $323 \mathrm{~K}$ at $\mathrm{pH}$ 9.0. Interestingly, it also retained relatively high activity in low- and high-temperature environments, displaying 40 and $20 \%$ of its maximum activity at 293 and $368 \mathrm{~K}$, respectively (Ding et al., 2014). Est 8 might be of significant industrial interest and value in scientific research as a thermostable esterase, and exhibits maximum activity in an alkaline environment. Moreover, Est8 can hydrolyze acetate at the $\mathrm{C} 3$ position of 7-aminocephalosporanic acid (7-ACA) to form deacetyl-7-ACA (D-7-ACA), as shown in Fig. 1 (data not shown). D-7-ACA is an important starting material for the production of semisynthetic $\beta$-lactam antibiotics (Levisson et al., 2012; Tian et al., 2014). To provide new insights into the structure-function relationship of Est8 and to obtain a better understanding of the biochemical data, determination of the crystal structure of the enzyme is in progress.

\section{Materials and methods}

\subsection{Macromolecule production}

The cloning, expression and purification of Est8, with the exception of gel filtration, have been described previously by Ding et al. (2014). Here, we describe the procedure in more detail. The gene encoding the enzyme was PCR-amplified from the genomic DNA of the thermophilic bacterium Bacillus sp. K91 using the primers 5'-GCAAATCATATTTA TCTTGC-3' (N-terminal) and $5^{\prime}$-CCTTTCTTTGATGATCG ATTC-3' (C-terminal). The PCR product was subsequently directly ligated with the pEASY-E2 expression vector according to the manufacturer's instructions. A hexahistidine tag (LEHHHHHH) was added from the vector sequence at the C-terminal end of the recombinant protein (Table 1). The ligation mixture was then transformed into Escherichia coli BL21 (DE3) cells (Novagen). Individual constructs were isolated from the transformants, screened for the correct size
Table 1

Macromolecule-production information.

\begin{tabular}{ll}
\hline Source organism & Bacillus sp. K91 \\
DNA source & Bacillus sp. K91 \\
Forward primer & 5'-GCAAACATATTTATCTTGC-3' \\
Reverse primer & 5'-CCTTTCTTTGATGATCGATTC-3' \\
Expression vector & pEASY-E2 \\
Expression host & E. coli BL21 (DE3) \\
Complete amino-acid sequence & MANHIYLADSTVQTYGDSTNQGGWGQFLG \\
of the construct produced & SHLPEHIQVINRAIGGRSSKTFVEEGL \\
& QAILDVIEPDDWLFVQMGHNDASKNKPE \\
& RYTEPYTTYKQYLKQYIAGAREKGAHPL \\
& LITPVARFHYNGVFLNDFPYCIAMKQ \\
& TAEEENVQLIDLMEKSLAFFTEKGEEKV \\
& YTYFMISEGINDYTHFTKKGANEMAKLV \\
& AKGIKELGLPLTESIIKERLEHHHHHH \\
\hline
\end{tabular}

Table 2

Crystallization.

\begin{tabular}{ll}
\hline Method & Hanging-drop vapour diffusion \\
Plate type & 16-well Linbro cell-culture plates \\
Temperature $(\mathrm{K})$ & 289 \\
Protein concentration & 12 \\
Buffer composition of protein solution & $20 \mathrm{~m} M$ Tris- $\mathrm{HCl}, 0.5 \mathrm{M} \mathrm{NaCl} \mathrm{pH} 7.2$ \\
Composition of reservoir solution & $2.0 \mathrm{M}$ ammonium sulfate, $5 \%(v / v)$ \\
& 2 -propanol \\
Volume and ratio of drop & $2 \mu \mathrm{l}, 1: 1$ \\
Volume of reservoir $(\mu \mathrm{l})$ & 200 \\
\hline
\end{tabular}

insert and completely sequenced to confirm their nucleotide identity. The transformed strains were grown in LB medium containing $100 \mathrm{mg} \mathrm{ml}^{-1}$ ampicillin at $310 \mathrm{~K}$ until the $\mathrm{OD}_{600}$ reached 0.6. Following induction with $0.7 \mathrm{~m} M$ isopropyl $\beta$-D-1thiogalactopyranoside (IPTG) at $293 \mathrm{~K}$ for $20 \mathrm{~h}$, the cells were harvested by centrifugation and disrupted by sonication. The lysate was centrifuged at $15000 \mathrm{~g}$ for $30 \mathrm{~min}$ and the supernatant was applied onto an $\mathrm{Ni}^{2+}$-NTA agarose-gel column for purification using a linear imidazole gradient of $20-500 \mathrm{~m} M$ in buffer $A(20 \mathrm{~m} M$ Tris-HCl, $0.5 M \mathrm{NaCl}$ pH 7.2). The target protein was eluted with buffer $A$ containing $80 \mathrm{~m} M$ imidazole. The obtained Est 8 protein was further purified by gel filtration using a Superdex 75 10/300 GL column (GE Healthcare) in running buffer consisting of $20 \mathrm{~m} M$ Tris- $\mathrm{HCl}, 0.5 \mathrm{M} \mathrm{NaCl} \mathrm{pH}$ 7.2. Finally, the pure Est8 protein was concentrated to 10 $15 \mathrm{mg} \mathrm{ml}^{-1}$ using an Amicon Ultra-15 centrifugal filter unit (10 000 nominal molecular-weight limit; Millipore) for crystallization.

\subsection{Crystallization}

Initial crystallization of Est8 was attempted by the hangingdrop vapour-diffusion method at $289 \mathrm{~K}$ using the Crystal

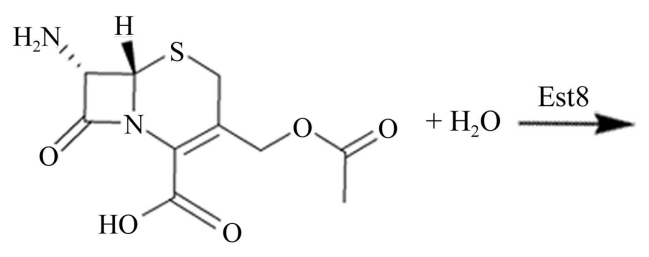

Figure 1

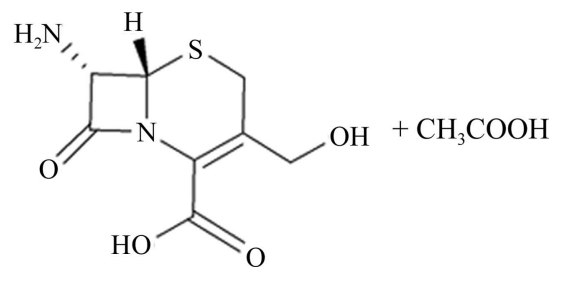

Reaction scheme for the esterase using 7-ACA as a substrate. 
Screen, Crystal Screen 2, PEG/Ion and SaltRx reagent kits (Hampton Research). $1 \mathrm{ml}$ protein solution was mixed with $1 \mathrm{ml}$ reservoir solution and the mixture was equilibrated against $200 \mathrm{ml}$ reservoir solution. Fusiform-shaped crystals were observed after $4 \mathrm{~d}$ using buffer consisting of $2.0 \mathrm{M}$ ammonium sulfate, $5 \%(v / v)$ 2-propanol. The crystallization conditions were optimized via variation of the ammonium sulfate concentration $(0.5-5.0 \mathrm{M})$, 2-propanol concentration $[2-8 \%(v / v)]$ and protein concentration (to a maximum of $15 \mathrm{mg} \mathrm{ml}^{-1}$ ) at $289 \mathrm{~K}$ in 16 -well plates (Table 2).

\subsection{Data collection and processing}

Before data collection, the crystals were soaked in mother liquor containing $20 \%(v / v)$ glycerol as a cryoprotectant and were flash-cooled directly in liquid nitrogen. Data sets were collected on beamline BL17U1 at the Shanghai Synchrotron Radiation Facility (SSRF), People's Republic of China using an ADSC Q315 CCD system (Wang et al., 2015). The exposure time was $1.0 \mathrm{~s}$ per frame. One complete data set was obtained by collecting 180 frames with $1^{\circ}$ oscillation. The data collected were processed and scaled with the HKL-2000 suite (Otwinowski \& Minor, 1997).

\section{Results and discussion}

Est8 from Bacillus sp. K91, with a calculated molecular mass of $24.5 \mathrm{kDa}$ and a theoretical isoelectric point of 6.45 , was cloned to contain a C-terminal $6 \times$ His tag, overproduced in E. coli BL21 (DE3) cells and purified to homogeneity in a two-step procedure by Ni-NTA affinity and size-exclusion

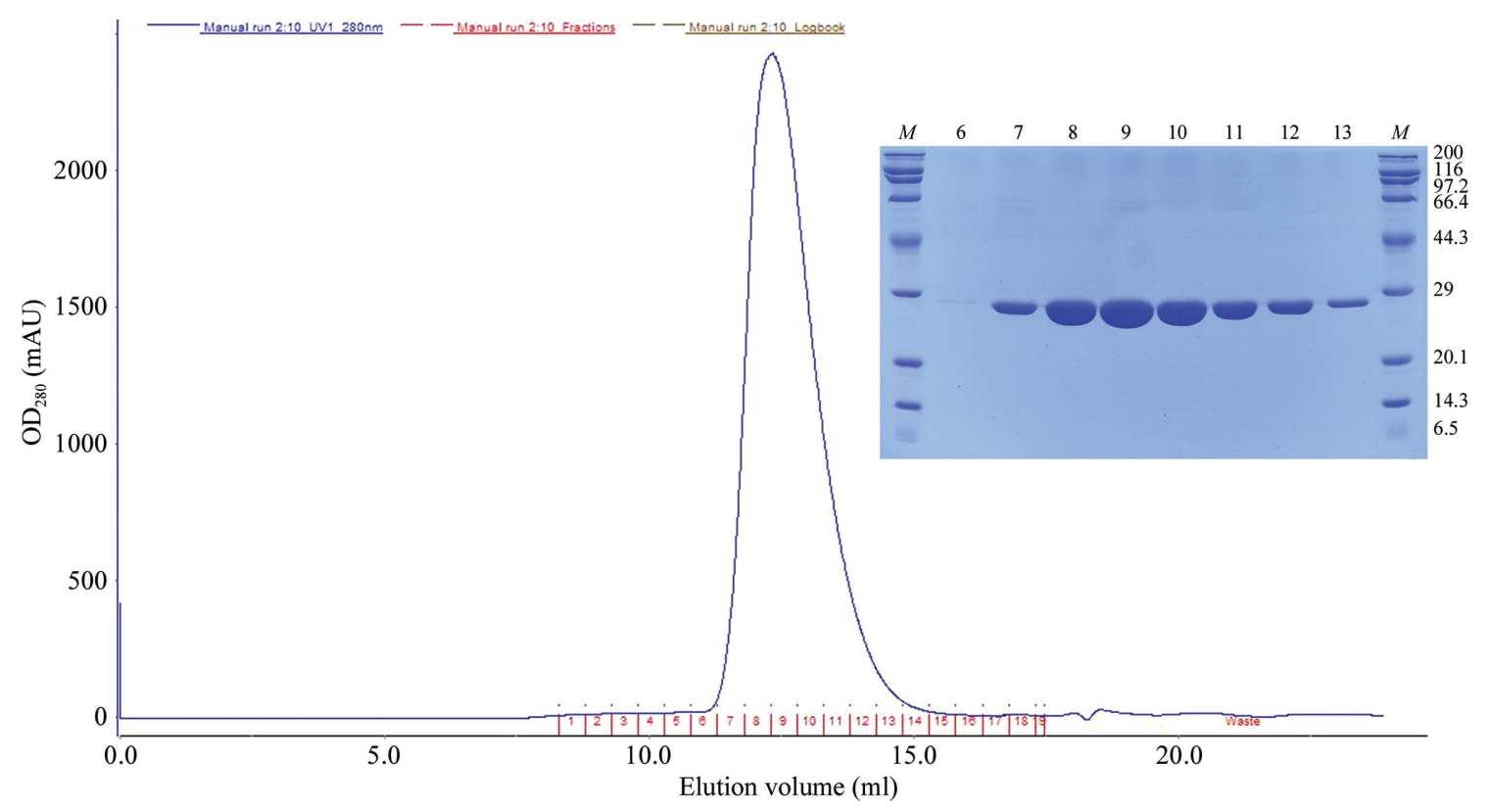

Figure 2

Size-exclusion chromatography and SDS-PAGE (inset) of Est8. In the SDS-PAGE, lane $M$ contains molecular-weight markers (labelled in kDa) and lanes 6-13 contain purified Est8 obtained at different volumes of elution buffer as shown on the size-exclusion chromatogram.

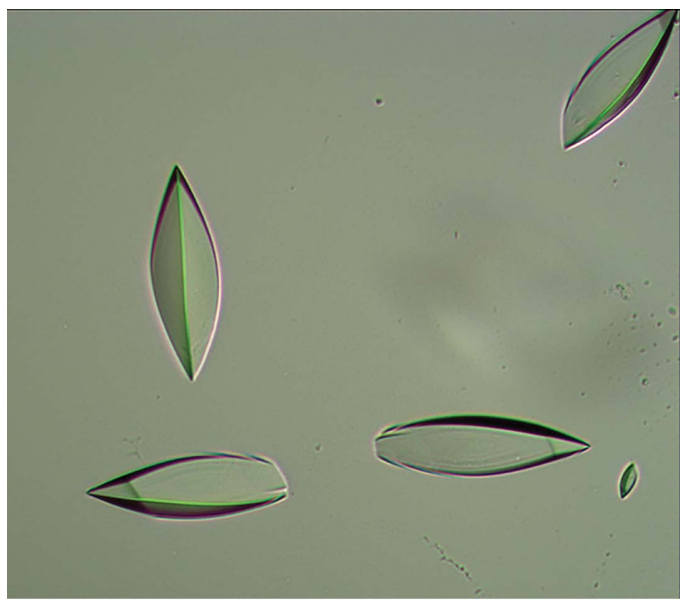

(a)

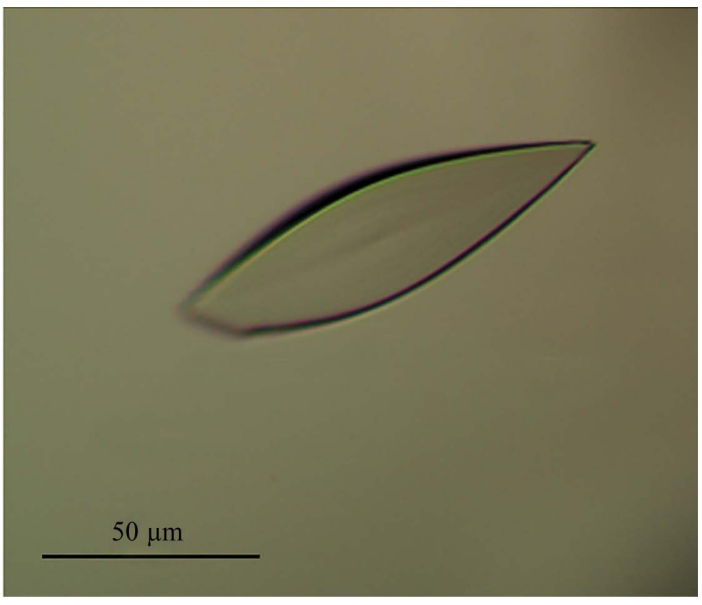

(b)

Figure 3

Pictures of Est8 protein crystals. (a) Initial crystallization screening with Est8 in $20 \mathrm{~m} M$ Tris- $\mathrm{HCl}, 0.5 M \mathrm{NaCl}$ pH 7.2 using the crystallization condition 2.0 M ammonium sulfate, $5 \%(v / v)$ 2-propanol. (b) Picture of an Est8 crystal. 
chromatography for crystallization (Fig. 2). The final purity of Est 8 was at least $99 \%$ as estimated by SDS-PAGE, and gel filtration indicated that it was monomeric. Significant enzyme activity was maintained after purification (Ding et al., 2014).

Purified recombinant Est8 enzyme, which can be stored at $277 \mathrm{~K}$ for several weeks without suffering protein degradation, was concentrated to $10-15 \mathrm{mg} \mathrm{ml}^{-1}$ before being subjected to crystallization. Initial crystallization screening showed that a reservoir solution consisting of $2.0 \mathrm{M}$ ammonium sulfate, $5 \%(v / v)$ 2-propanol could be used to grow Est8 crystals. Attempts were made to optimize the crystallization conditions for Est8 by varying the concentration of ammonium sulfate $(0.5-5.0 \mathrm{M})$ and 2-propanol [4-7.5\% $(v / v)]$ based on the initial crystallization conditions. Crystals could be grown under conditions consisting of $2.0 \mathrm{M}$ ammonium sulfate with 2-propanol at a concentration ranging from 4 to $7.5 \%(v / v)$. Thus, sufficiently large crystals were obtained both with the initial condition from the kit and using optimized conditions (Fig. 3a). The dimensions of the crystals were approximately $0.05 \times 0.05 \times 0.1 \mathrm{~mm}$ (Fig. $3 b$ ). The crystals diffracted to $2.30 \AA$ resolution and belonged to space group $P 4_{1} 2_{1} 2$ or $P 4_{3} 2{ }_{1} 2$, with unit-cell parameters $a=b=68.50, c=79.57 \AA$ (Fig. 4). Assuming the presence of one molecule in the asymmetric unit, the Matthews coefficient $V_{\mathrm{M}}$ was calculated to be $1.90 \AA^{3} \mathrm{Da}^{-1}$, with a solvent content of approximately $35.2 \%$. The statistics of data collection are summarized in Table 3. Initial phase determination was carried out by molecular replacement with Phaser using the crystal structure of the hypothetical YxiM precursor from B. subtilis (PDB entry 2o14, chain $A$; Northeast Structural Genomics

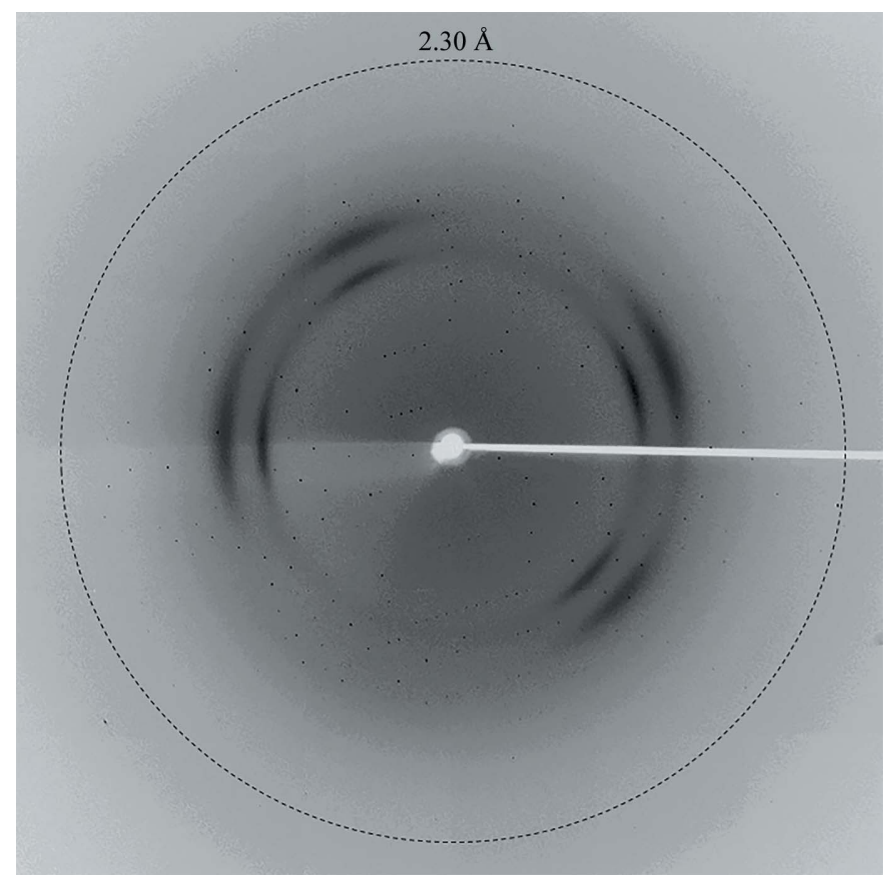

Figure 4

A typical X-ray diffraction pattern collected from an Est8 protein crystal. The diffraction image was collected on beamline BL17U1 at SSRF using an ADSC Q315 CCD detector. The detector edge corresponds to $\sim 2.0 \AA$ resolution.
Table 3

Data collection and processing.

\begin{tabular}{ll}
\hline Diffraction source & Beamline BL17U1, SSRF \\
Wavelength $(\AA)$ & 0.9792 \\
Temperature $(\mathrm{K})$ & 100 \\
Detector & ADSC Q315 CCD \\
Crystal-to-detector distance $(\mathrm{mm})$ & 300 \\
Rotation range per image $\left({ }^{\circ}\right)$ & 1 \\
Total rotation range $\left({ }^{\circ}\right)$ & 180 \\
Exposure time per image (s) & 1 \\
Space group & $P 4_{1} 2_{2} 2$ or $P 4_{3} 2_{1} 2$ \\
Unit-cell parameters $(\AA)$ & $a=b=68.50, c=79.57$ \\
Resolution range $(\AA)$ & $50-2.30(2.38-2.30)$ \\
Total No. of reflections & 54346 \\
No. of unique reflections & $8899(858)$ \\
Completeness $(\%)$ & $100(100)$ \\
Multiplicity & $6.1(6.3)$ \\
$R_{\text {merge }} \dagger(\%)$ & $16.4(60.5)$ \\
$V_{\mathrm{M}}\left(\AA^{3} \mathrm{Da}{ }^{-1}\right)$ & 1.90 \\
Mean $I / \sigma(I)$ & $14.8(3.3)$ \\
Overall $B$ factor from Wilson plot $\left(\AA^{2}\right)$ & 15.9 \\
\hline
\end{tabular}

$\dagger R_{\text {merge }}=\sum_{h k l} \sum_{i}\left|I_{i}(h k l)-\langle I(h k l)\rangle\right| / \sum_{h k l} \sum_{i} I_{i}(h k l)$, where $\langle I(h k l)\rangle$ is the mean intensity of the observations $I_{i}(h k l)$ of reflection $h k l$.

Consortium, unpublished work) as a search model. This protein belongs to the SGNH hydrolase family, as does Est8, and displayed 35\% identity to Est8. Owing to the low aminoacid sequence identity with Est8, attempts to solve the structure of Est 8 using molecular replacement failed. Therefore, the preparation of selenomethionyl-derivatized Est8 protein and heavy-atom soaking is currently under way for the structure determination of the GDSL-family thermostable esterase Est8. Once structural information for Est8 has been obtained, it will be used as a starting point to better understand its catalytic mechanism and potential applications in industry.

\section{Acknowledgements}

We thank the staff of Shanghai Synchrotron Radiation Facility beamline BL17U1 for assistance in data collection. We gratefully acknowledge Professor Yanhui Xu from Fudan University, People's Republic of China for help with Est8 structure determination, structure analysis and paper revision.

\section{Funding information}

Funding for this research was provided by: National Natural Science Foundation of China (grant Nos. 31460022 and 31600083); Natural Science Foundation of Yunnan Province (grant No. 2016FB036).

\section{References}

Akoh, C. C., Lee, G.-C., Liaw, Y.-C., Huang, T.-H. \& Shaw, J.-F. (2004). Prog. Lipid Res. 43, 534-552.

Bornscheuer, U. T. (2002). FEMS Microbiol. Rev. 26, 73-81.

Ding, J. M., Yu, T. T., Liang, L. M., Xie, Z. R., Yang, Y. J., Zhou, J. P., Xu, B., Li, J. J. \& Huang, Z. X. (2014). J. Microbiol. Biotechnol. 24, $1551-1558$.

Hess, M., Katzer, M. \& Antranikian, G. (2008). Extremophiles, 12, 351-364.

Hotta, Y., Ezaki, S., Atomi, H. \& Imanaka, T. (2002). Appl. Environ. Microbiol. 68, 3925-3931.

Jaeger, K. E. \& Eggert, T. (2002). Curr. Opin. Biotechnol. 13, 390-397. 
Levisson, M. et al. (2012). Proteins, 80, 1545-1559.

López-López, O., Cerdán, M. E. \& González Siso, M. I. (2014). Curr. Protein Pept. Sci. 15, 445-455.

Mølgaard, A., Kauppinen, S. \& Larsen, S. (2000). Structure, 8, $373-$ 383.

Otwinowski, Z. \& Minor, W. (1997). Methods Enzymol. 276, 307-326. Rubin, B. (1994). Nature Struct. Biol. 1, 568-572.
Stergiou, P. Y., Foukis, A., Filippou, M., Koukouritaki, M., Parapouli, M., Theodorou, L. G., Hatziloukas, E., Afendra, A., Pandey, A. \& Papamichael, E. M. (2013). Biotechnol. Adv. 31, 18461859.

Tian, Q. Q., Song, P., Jiang, L., Li, S. \& Huang, H. (2014). Appl. Microbiol. Biotechnol. 98, 2081-2089.

Wang, Q.-S. et al. (2015). Nucl. Sci. Tech. 26, 010102. 